\title{
RELATIVISTIC FLUID SPHERES
}

\author{
R.R. KING
}

Static spherically symmetric solutions of Einstein's field equations for a perfect fluid have found application as relativistic models for stars which are in mechanical and thermodynamical equilibrium.

In either Schwarzschild (canonical) or isotropic coordinate systems, a spherically symmetric metric gives rise to three non-linear second order differential equations. The standard problem is to find analytic forms for the two potentials of the metric, subject to the usual physical constraints: for a regular sphere, both the pressure and density must be positive and monotonic decreasing, and there must follow a realistic equation of state, preferably in simple closed form. Boundary conditions are derived from the requirement that any solution join continuously to the Schwarzschild solution for free space at the boundary of the sphere.

To guarantee a result which is physically valid, some authors have assumed a subsidiary relationship, for example, an equation of state. An ensuing difficulty is then, however, that such an assumption may not lead necessarily to equations which can be solved. The more usual approach has been to use the large body of theory of differential equations to introduce mathematical simplifications and hope that a reasonable equation of state will emerge.

This thesis demonstrates initially that often the same solutions keep re-appearing in the literature without proper identification of previous discoveries having been made. It then shows that the field equations can be integrated, subject to the application of two different ansatz, provided that, for each ansatz, a single integral of generalized form and involving

Received 21 February 1983. Thesis submitted to University of New England October 1981. Degree approved April 1983. Supervisor: Dr N.W. Taylor. 
two parameters, can be evaluated. In most solution schemes, two integrations are required and their replacement by a single one, the possibility (or otherwise) of which is immediately evident, constitutes an improved solution process. Expressions for pressure and density follow immediately, although there is not necessarily a simple closed relationship between these quantities.

Several quadratures for the case of each ansatz have been completed. For one of these, values of the parameters in the integral yield a solution of physical relevance similar to, although more complex than, already known ones. An equation of state can be obtained when limiting conditions apply, and the predictions of the model for the mass, radius and central density of a neutron star accord reasonably well with accepted values.

\section{References}

[1]. H. Heintzmann, "New exact static solutions of Einstein's field equations", 2. Physik 228 (1969), 489-493.

[2] D. Kramer, H. Stephani, E. Herlt, M. MacCallum, Exact solutions of Einstein's field equations (Cambridge University Press, Cambridge, London, New York, 1980).

[3] Richard C. Tolman, "Static solutions of einstein's field equations for spheres of fluid", Phys. Rev. 55 (1939), 364-373.

St Patrick's College,

Private Bag,

Strathfield,

New South Wales 2135 ,

Australia. 